\title{
Generalization of the Rosen-Zener Model of Noncrossing Interactions. II. Differential Cross Sections.
}

T. R. Dinterman

John B. Delos

William \& Mary, jbdelos@wm.edu

Follow this and additional works at: https://scholarworks.wm.edu/aspubs

Part of the Physics Commons

\section{Recommended Citation}

Dinterman, T. R. and Delos, John B., Generalization of the Rosen-Zener Model of Noncrossing Interactions. II. Differential Cross Sections. (1977). Physical Review A, 15(2), 475-478.

https://doi.org/10.1103/PhysRevA.15.475

This Article is brought to you for free and open access by the Arts and Sciences at W\&M ScholarWorks. It has been accepted for inclusion in Arts \& Sciences Articles by an authorized administrator of W\&M ScholarWorks. For more information, please contact scholarworks@wm.edu. 


\title{
Generalization of the Rosen-Zener model of noncrossing interactions. II. Differential cross sections*
}

\author{
T. R. Dinterman and J. B. Delos \\ Department of Physics, College of William and Mary, Williamsburg, Virginia 23184
}

(Received 15 May 1975)

\begin{abstract}
The generalized Rosen-Zener model developed in the accompanying paper is used to calculate differential cross sections for a model noncrossing system, and the structure of the cross sections is explained in terms of the deflection functions. It is found that the angular threshold effects that are typical of potential-curve crossings do not appear in this noncrossing system.
\end{abstract}

\section{GENERAL CONSIDERATIONS}

Measurements of differential cross sections as a function of velocity and scattering angle are very sensitive to the detailed dynamics of the collision process, and one of the objectives of such experiments is to obtain information about the kinds of interactions and the nature of the potential curves involved in a given collision. Accordingly, it is useful to examine general model systems to find what kinds of features might be expected in a differential cross section, and how these features can be interpreted.

In a previous paper, ${ }^{1}$ we have carried out such calculations for a "typical" curve crossing, and we showed that a number of phenomena can normally be expected. For example, if the potential curves are repulsive, the (1-1) elastic differential cross section can be expected to be smooth at small $E \theta$ and oscillatory at large $E \theta$; at an $E \theta$ corresponding to the crossing region, there may be two rainbows, and between them would be a complicated three-term interference pattern.

In order to find out whether the same sorts of phenomena would also occur for noncrossing interactions, we have carried out calculations of the differential cross section for some noncrossing situations.

There are two general types of noncrossing interactions. In the first case, two potential curves may approach each other closely and become degenerate in the limit of zero internuclear separation; the coupling is then usually a smoothly varying function of distance outside the innermost classical turning point. Such a situation occurs in low-energy proton-hydrogen-atom collisions, in which a $\Sigma$ and a $\pi$ state are coupled by the Coriolis interaction. Differential cross sections for this system have been calculated by Knudson and Thorson. ${ }^{2}$ Since in this case, transitions are only likely near the turning point, the system will follow one potential curve on the incoming and another on the outgoing part of the trajectory; for repulsive potential curves there is then a 1-1 relationship between scattering angle and impact parameter, and so no special interference or rainbow phenomena result from the inelastic coupling. (See, for example, Fig. 6 of Ref. 2.)

In the second case, the potential curves may be relatively slowly varying, but the coupling between them rapidly increases. This is the situation that occurs in alkali-ion-atom charge exchange, as discussed in the preceding paper. ${ }^{3}$ In this case, since transitions occur mainly near $R_{c}$, the system can make a transition either on the incoming or the outgoing part of the trajectory. Then two impact parameters may lead to the same final scattering angle, and an interference pattern may appear in the differential cross section. It is this situation that is considered in the following sections.

\section{FORMULAS AND METHOD OF CALCULATION}

The formulas needed for this calculation have been presented in Ref. 1 and 3. The scattering amplitude is obtained from

$$
f_{m n}=\left(2 i k_{i}\right)^{-1} \sum_{L}(2 L+1) P_{L}(\cos \theta)\left(S_{m n}^{L}-\delta_{m n}\right),
$$

where $k_{i}$ is the magnitude of the wave vector associated with the initial state. The $S$ matrix is obtained from Eqs. (7) and (4) of the preceding paper, ${ }^{3}$ with $\underline{G}_{+}$given in Eq. (12) or (A12) and (A13). There are a total of six important scattering angles, two each for elastic scattering in the ground state, elastic scattering in the excited state, and inelastic scattering; these are given by Eq. (6) of Ref. 1.

The formulas for $G_{+}$obtained in the preceding paper refer to the diabatic representation, so 
these formulas should be combined with diabatic phase shifts to obtain $S$. However, when the calculation is performed this way, it is found that $Z, \Gamma_{1}$, and $\Gamma_{2}$ are rapidly oscillating functions of the impact parameter. As a consequence, they do not provide a simple physical interpretation of the scattering process, and it is not useful to consider them. However, there is an alternative procedure.

The formulas for $G_{+}$can be transformed into the adiabatic representation, using the inverse of Eqs. (59) and (60) of Ref. 4, and the resulting adiabatic $G_{+}$combined with adiabatic phase shifts. Although the cross section must not depend upon the representation used in the calculation, the physical interpretation of the cross section may be easier in one or the other representation. We have found that for some reason the adiabatic representation is to be preferred for these calculations, because the adiabatic $G$-matrix parameters are smooth and well-behaved and easy to interpret. This also holds in curve-crossing problems, as was shown in Ref. 1.

\section{THE MODEL}

A reasonable set of model potentials were chosen, and they are shown in Fig. 1. Their analytic forms are (diabatic representation)

$$
\begin{aligned}
& V_{11}=A e^{-R / R_{a}}+B e^{-R / R_{b}}, \\
& V_{22}=V_{11}+E_{2}^{0}, \\
& V_{12}=D e^{-R / R_{d},}
\end{aligned}
$$

with (Hartree units)

$$
\begin{aligned}
& A=1.152, \quad R_{a}=1.38, \\
& B=1.864, \quad R_{b}=0.30, \quad E_{2}^{0}=0.00925, \\
& D=1.864, \quad R_{d}=3.00 .
\end{aligned}
$$

The energy for this calculation was

$$
E=0.35 \text { hartree }=9.52 \mathrm{eV},
$$

and the mass was taken to be that of a proton $\left(1836 m_{e}\right)$.

Since we were especially interested in the effects that occur near the angular threshold for inelastic scattering, we have used method 2 of the preceding paper, ${ }^{3}$ i.e., choosing the model $t(s)$ $=[\alpha \cos \zeta s]^{-1}$, so that it matches the actual $t(s)$ as closely as possible at the turning point. Thus, for each $L, t$, and $d^{2} t / d s^{2}$ were evaluated at the turning point using Eq. (18), and $\alpha$ and $\zeta$ were evaluated from Eq. (19) of the preceding paper. ${ }^{3}$ The diabatic $G_{+}$matrix was evaluated using Eqs. (A12), (A13), and (A5) of that paper, and it was transformed into the adiabatic representation using the inverse of Eq. (60) of Ref.4. Then the $\delta$

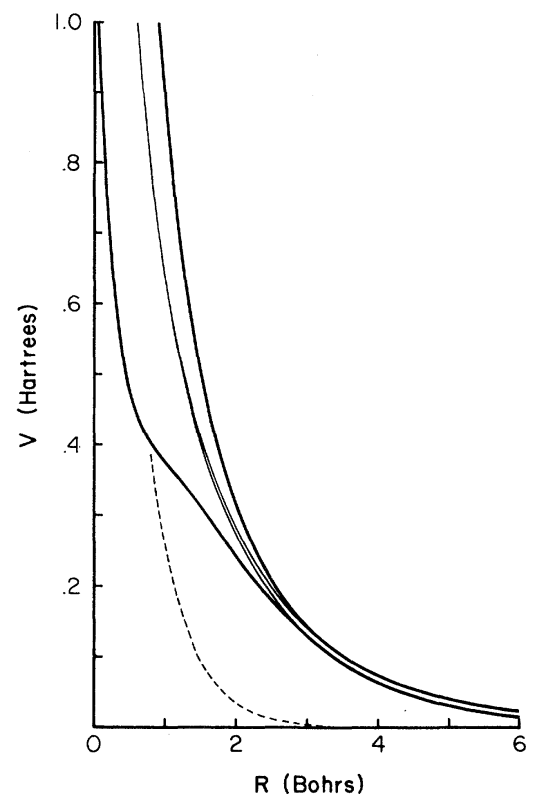

FIG. 1. Interatomic potentials of Eq. (2). Light lines: diabatic representations. The difference between $V_{11}$ and $V_{22}$ is constant, and the coupling (dashed line) rapidly decreases with increasing $V_{12}$. Heavy lines: adiabatic representation, obtained by diagonalizing $\underline{V}$.

matrix was evaluated from (7) of Ref. 3, using the adiabatic phase shifts and the adiabatic $G_{+}$, and it was used in Eq. (1) of the present paper.

We have mentioned that simple models for $t(s)$ like the one used here often do not accurately reproduce the interference patterns that appear in differential (and sometimes in total) cross sections. This is not a major difficulty: the pattern can be obtained quantitatively by modifying the argument of the $\sin ^{2}$ factor in the transition probability (see Ref. 1 for an example of this). However, since we are mainly interested in the qualitative features of the differential cross section, and because the phase should be accurate enough in the threshold region to reproduce these features, we have made no attempt to modify it.

\section{RESULTS}

The transition probability halfway through the collision, $Z^{2}$, is shown in Fig. 2 as a function of $L$. At small $L$, the system makes a transition from the lower adiabatic curve to the upper one about $15 \%$ of the time; obviously this probability goes to zero at large $L$. It is interesting, however, that the decline with $L$ is smooth and gradual; this is in sharp contrast to its behavior for curve crossings, where an abrupt drop is found at the threshold. (Note on the other hand that the spuriously long-range behavior obtained by Melius 


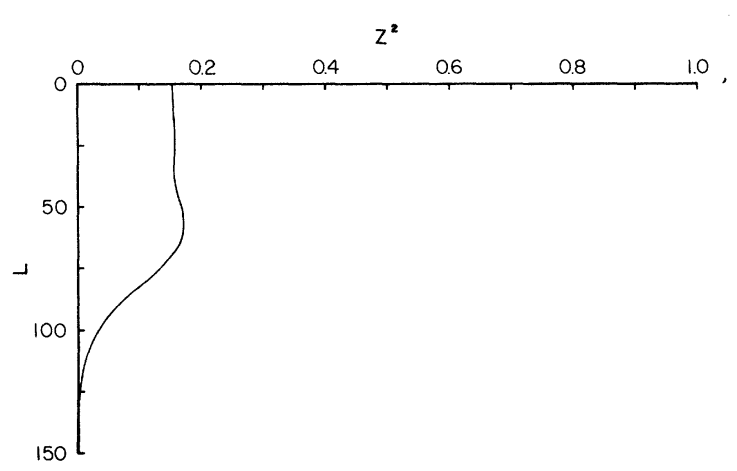

FIG. 2. Transition probability half way through collision, $Z^{2}$, in adiabatic representation vs angular momentum $L$.

and Goddard ${ }^{5}$ from the Rapp-Francis formula does not appear.)

In Fig. 2 are shown the scattering angles for collisions that begin and end in the ground state, and the corresponding differential cross section. At large $L$ no excitations are possible, and there is only one possible scattering angle. At small $L$, the system can stay entirely on the lower adiabatic, or make a transition to the upper one on the way in and back to the lower one on the way out; hence there are two possible scattering angles. At small angles, the 1-1 differential cross section is characteristic of repulsive potentials; at about $25^{\circ}$ the elastic cross section begins to be depleted by the inelastic process, and above $45^{\circ}$ an interference pattern appears. But the interesting thing about these curves is that they show none of the spectacular effects that may be found in curve crossings near the angular threshold (see, for example, Fig. 4 of Ref. 1).

For elastic scattering that begins and ends in the upper state, since the diabatic potentials differ so little (their splitting is much less than the collision energy), the 2-2 cross section is almost identical to the 1-1 cross section.

And finally, again because of the small energy defect, the scattering angles for excitation or deexcitation are essentially the same as the elastic scattering angles. The cross section, of course, differs from the elastic cross section because of the transition probability: $\sigma_{12}(\theta)$ is small at small angles (though it falls off rather more gradually than in the curve-crossing case) and its oscillations are necessarily out of phase with the oscillations in $\sigma_{11}$ or $\sigma_{22}$.

\section{DISCUSSION}

It is not surprising that the threshold effects that may be so prominent in curve crossings do not appear here. In a curve crossing, especially if the
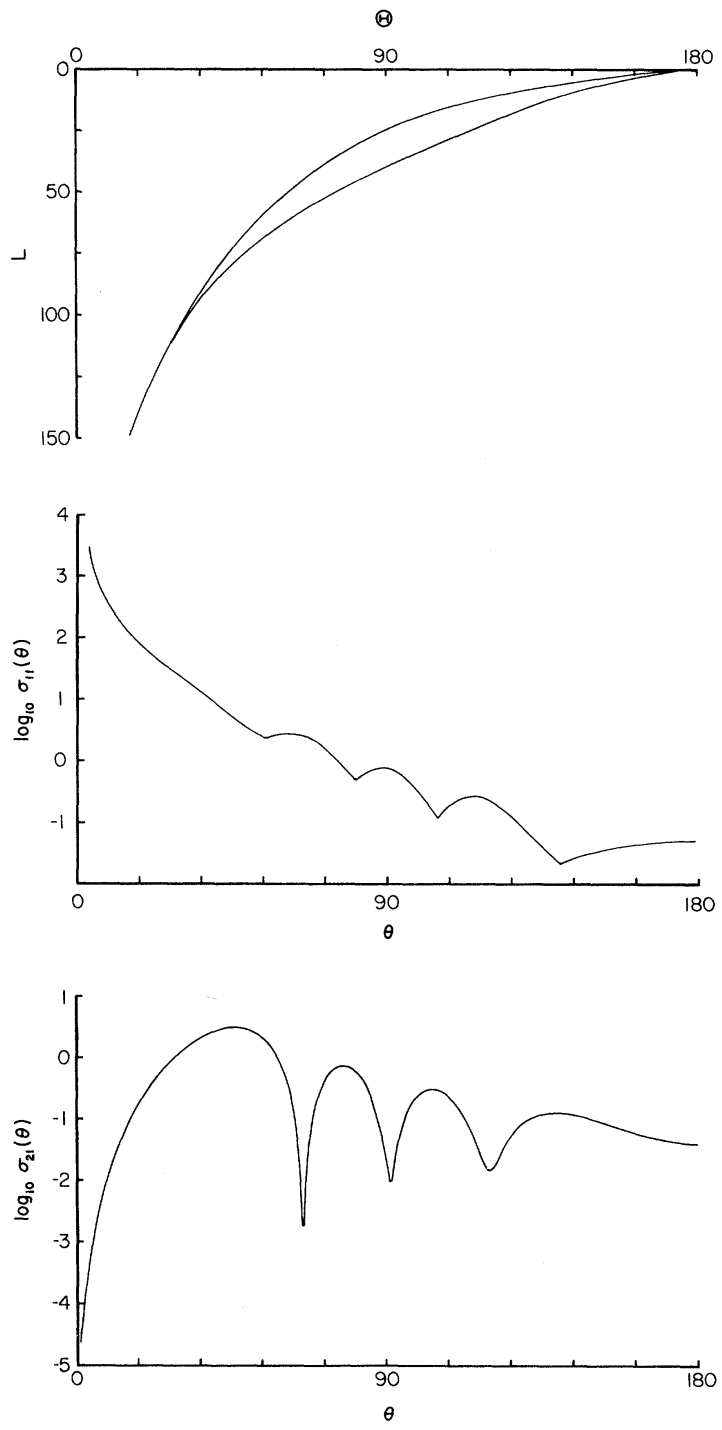

FIG. 3. Top: Deflection functions for ground state to ground-state $(1-1)$ collisions, in adiabatic representation. Middle: $\log _{10}$ [elastic $(1 \rightarrow 1)$ scattering cross section]. Bottom: $\log _{10}$ [inelastic $(2 \rightarrow 1)$ scattering cross section].

coupling is weak ( $V_{12}$ is small), the change in character of the electronic states may be very abrupt, while in the present case, this change is more smooth and gradual. We have varied the assumed potentials within reasonable limits and in no case have we found any of the sudden changes, or the associated threshold effects that are common in curve crossings. This does not mean that such threshold effects can not occur in noncrossing situations, but only that they usually will not occur. (For that matter, they don't necessarily occur in curve crossings either.)

Approximate formulas for the deflection functions 
display more clearly the difference between these two cases. For (1-1) scattering (elastic scattering on the ground state) the difference between the two deflection functions is given from Eq. (6) of Ref. 1:

$$
\Theta_{1}-\Theta_{2}=2 \frac{d}{d L}\left(\Gamma_{1}+\Gamma_{2}\right)
$$

For curve crossings, the simplest possible approximation to the inelastic phases gives

$$
\Gamma_{1}+\Gamma_{2} \simeq \int_{R_{0}}^{R_{x}} \frac{V_{22}-V_{11}}{\hbar v(R)} d R+\text { (corrections). }
$$

Ignoring the corrections, for $R_{0}$ (the turning point) close to $R_{x}$ (the crossing point), we may use a linear potential and constant force approximation to obtain

$$
\begin{aligned}
& v(R) \simeq\left(\frac{2 F}{M}\left(R-R_{0}\right)\right)^{1 / 2}, \\
& \Gamma_{1}+\Gamma_{2} \propto\left(R-R_{0}\right)^{3 / 2},
\end{aligned}
$$

and therefore,

$$
\Theta_{1}-\Theta_{2} \propto\left(L_{x}-L\right)^{1 / 2},
$$

where $L_{x}$ is the angular momentum such that $R_{0}$ $=R_{x}$. This means that the two angles diverge from each other with infinite slope; Olson and Smith ${ }^{6}$ have shown that if this were rigorously valid, it would guarantee the presence of a minimum in $\Theta_{1}$, and a corresponding rainbow in the cross section; since (5) is usually approximately true, it means that threshold rainbows will usually appear for curve crossings.

For the noncrossing case, a different approximation to $\Gamma_{1}+\Gamma_{2}$ is appropriate. Comparing Eqs. (6) and (13) of the preceding paper, it follows that
$\Gamma_{1}+\Gamma_{2}$ is given approximately by

$$
\Gamma_{1}+\Gamma_{2} \simeq \pi / \zeta
$$

which, using Eq. (28) of that paper, becomes

$$
\Gamma_{1}+\Gamma_{2} \propto \exp (-L \times \text { const }),
$$

and likewise for $\Theta_{1}-\Theta_{2}$. The two angles therefore diverge from each other smoothly; the abrupt change that leads to maxima and minima in the scattering angles does not occur, and the threshold rainbows do not normally occur in a noncrossing situation.

The same result can also be obtained by calculation of the classical deflection functions in the adiabatic representation ${ }^{7}$ : the rainbows that occur in noncrossing problems will usually be associated with minima in the adiabatic potential curves, not with the critical region of electronic excitations.

\section{CONCLUSION}

We have used one of the forms $\mathrm{s}^{8}$ of the generalized Rosen-Zener model to calculate the differential cross section for a simple two-state system having potential curves which do not cross. The resulting scattering angles and differential cross sections are shown in Fig. 3. The angular threshold appears at a fairly large angle because the potential curves in our model system are strongly repulsive; attractive potentials will modify the scattering angles and cross sections in the obvious way, leading to rainbow phenomena and a more complicated interference pattern. However the qualitative features of the transition probability and $\Theta_{1}-\Theta_{2}$ should be the same; in particular the special threshold rainbows that usually appear in a curve crossing system should not normally appear in a noncrossing system.
*Based in part upon an Honors Thesis submitted by T. R. D. for a B. S. in Physics, College of William and Mary, 1974.

${ }^{1} J$. B. Delos, Phys. Rev. A 9, 1626 (1974). See also S. M. Bobbio, L. D. Doverspike, and R. L. Champion, Phys. Rev. A $\underline{7}, 526$ (1973).

${ }^{2}$ S. K. Knudson and W. R. Thorson, Can. J. Phys. $\underline{48}$, 313 (1970).

${ }^{3}$ T. R. Dinterman and J. B. Delos, preceding paper, Phys. Rev. A 15, 463 (1977).

${ }^{4}$ J. B. Delos and W. R. Thorson, Phys. Rev. A $\underline{6}, 728$ (1972).
${ }^{5}$ C. F. Melius and W. A. Goddard, Phys. Rev. A 10, 1541 (1974).

${ }^{6}$ R. E. Olson and F. T. Smith, Phys. Rev. A $\underline{3}, 1607$ (1971).

${ }^{7}$ The curve-crossing case is treated this way by R. P. Saxon and R. E. Olson, Phys. Rev. A 12, 830 (1975).

${ }^{8}$ The form of the model that we used here (called method II in Ref. 3) seems most appropriate for the study of phenomena associated with turning points. However, the other forms of the model would also give results that are qualitatively similar. 